\section{Jornal de Pediatria}

Lahorgue Nunes, Magda; Bruni, Oliviero

A genética dos distúrbios do sono na infância e adolescência Jornal de Pediatria, vol. 84, núm. 4, julio-agosto, 2008, pp. S27-S32

Sociedade Brasileira de Pediatria

Porto Alegre, Brasil 


\title{
The genetics of sleep disorders in childhood and adolescence
}

\author{
A genética dos distúrbios do sono na infância e adolescência \\ Magda Lahorgue Nunes ${ }^{1}$, Oliviero Bruni
}

\section{Resumo}

Objetivo: $\mathrm{O}$ objetivo deste artigo é revisar a literatura sobre a genética dos distúrbios do sono na infância e adolescência.

Fontes dos dados: As palavras-chave "sono" e "genética" foram usadas para pesquisar por artigos publicados nos últimos cinco anos no banco de dados MEDLINE. A seguir, seus resumos foram analisados. A pesquisa também incluiu artigos clássicos, com a primeira descrição dos genes.

Síntese dos dados: A recorrência familiar de muitos distúrbios do sono é um achado freqüente, mas loci genéticos foram descobertos para poucos deles. Descrevemos aqui distúrbios do sono transmitidos por herança genética e também aqueles que apresentam altos índices de recorrência familiar, apesar de nenhum gene específico haver sido encontrado.

Conclusões: Apesar da maioria dos distúrbios do sono ainda não terem uma base molecular identificada, técnicas modernas são cada vez mais utilizadas para determinar a contribuição dos genes ao sono e aos seus distúrbios associados. A importância clínica destas descobertas pode estar relacionada com a melhoria de métodos diagnósticos, mas também como alvo para o desenvolvimento de medicações específicas.

J Pediatr (Rio J). 2008;84(4 Supl):S27-32: Sono, genética, narcolepsia, síndrome das pernas inquietas, parassônias, infância.

\section{Introdução}

Os distúrbios do sono (DS) são relatados freqüentemente durante a infância e adolescência. A história familiar positiva é comum ${ }^{1}$. Devido a sua elevada prevalência podem representar uma epidemia mundial emergente ${ }^{2}$.

A importância do diagnóstico e tratamento dos distúrbios do sono em crianças depende, entre outros fatores, dos achados sobre a influência do sono e do temperamento na vida escolar ${ }^{3}$.

Em contraste com o progresso impressionante da genética molecular dos ritmos circadianos, pouco se sabe sobre a

\author{
Abstract \\ Objective: To review the literature regarding the genetics of \\ sleep disorders in childhood and adolescence.
}

Sources: Articles published in the past 5 years were searched on MEDLINE using the keywords sleep and genetics. Abstracts were then analyzed. Classical articles with the first description of genes were also included.

Summary of the findings: We often find familial recurrence in many sleep disorders. However, gene loci were discovered for only a few of them. We describe sleep disorders transmitted by genetic heritance and also those in that, although a gene was not found, familial recurrence is high.

Conclusion: Although most of the sleep disorders do not have by now an identified molecular basis, modern techniques are being increasingly applied to determine the contribution of genes to sleep and its associated disorders. The clinical importance of these discoveries may relate not only to improving diagnostic methods but also as a target for drug development.

J Pediatr (Rio J). 2008;84(4 Suppl):S27-32: Sleep, genetics, narcolepsy, restless leg syndrome, parasomnias, childhood.

base molecular do sono normal ${ }^{4-7}$. A pergunta sobre qual seria a função do sono segue sem resposta na Biologia ${ }^{5}$.

Muitos distúrbios do sono apresentam recorrência familiar, mas até hoje a pesquisa sobre a genética molecular dos distúrbios do sono continua sendo, surpreendentemente, um dos últimos campos ativos ${ }^{2}$.

Os estudos genéticos geralmente se restringem à narcolepsia, à síndrome das pernas inquietas (SPI) e a apnéia obstrutiva do sono.

Nos últimos anos, muitos genes foram associados a muitas doenças. O mapeamento destas alterações facilitou a identificação de pessoas afetadas, melhorou a compreensão de

1. MD, PhD. Associate professor, Faculdade de Medicina, Pontifícia Universidade Católica do Rio Grande do Sul (PUCRS), Porto Alegre, RS.

2. MD. Assistant professor, Center for Pediatric Sleep Disorders, Department of Developmental Neurology and Psychiatry, Faculty of Medicine, Sapienza University, Rome, Italy.

Apoio financeiro: O primeiro autor recebe apoio do CNPq (processo no 300742 -2007).

Não foram declarados conflitos de interesse associados à publicação deste artigo.

Como citar este artigo: Nunes ML, Bruni O. The genetics of sleep disorders in childhood and adolescence. J Pediatr (Rio J). 2008 ;84(4 Suppl):S27-32.

doi:10.2223/JPED.1786 
seu prognóstico e funcionou como novos alvos para o desenvolvimento de medicações.

O objetivo deste artigo é revisar a literatura sobre a genética dos distúrbios do sono na infância e adolescência para permitir a melhor compreensão destes distúrbios e para orientar o pediatra generalista em direção ao seu diagnóstico e prognóstico.

\section{Metodologia}

Foi realizada revisão da literatura sobre o tema no banco de dados MEDLINE, usando-se uma combinação das palavraschave "sono" e "genética", e a seguir uma busca isolada pelas palavras-chave "narcolepsia", "apnéia do sono" e "síndrome das pernas inquietas". Os resumos foram analisados e os artigos que continham informações sobre a faixa etária pediátrica foram incluídos. A pesquisa também incluiu artigos clássicos, com a primeira descrição dos genes envolvidos em DS.

\section{Resultados}

\section{Narcolepsia}

A narcolepsia é um distúrbio crônico do sono que ocorre com predominância levemente maior em indivíduos do sexo masculino, entre a infância e a quinta década de vida, atingindo seu clímax na segunda década. A doença é caracterizada por uma tétrade de sintomas relativos à ocorrência de sono REM (sono com movimento rápido dos olhos) em momentos inadequados: sonolência diurna excessiva, cateplexia (queda abrupta com perda de tônus muscular, sem perda de consciência, geralmente provocada por emoções como riso, raiva ou surpresa), paralisia do sono (experiência aterradora que ocorre ao início do sono ou ao acordar, em que o paciente se descobre subitamente incapaz de se mover, falar ou respirar profundamente) e alucinações hipnagógicas/ hipnopômpicas (sonhos vívidos que podem ser difíceis de distinguir da realidade e alucinações auditivas, visuais ou cenestopáticas que ocorrem no começo do sono, tanto diurno como noturno $)^{8,9}$.

A ocorrência familiar de narcolepsia-cataplexia foi relatada pela primeira vez em 1877 , por Westphal ${ }^{8}$. No entanto, a observação da associação da narcolepsia com o antígeno do leucócito humano (HLA) DR2 foi relatada pela primeira vez no Japão, cem anos depois ${ }^{8}$. Mais de $85 \%$ de todos os narcolépticos com cataplexia definida compartilham de uma mutação (um alelo de HLA) no cromossomo 6, seja apenas no HLA DQB1*0602 ou em conjunto com HLA DR15. Mais tarde, em 1999, um segundo gene, a orexina (hipocretina), localizado no cromossomo 12, foi relacionado com a narcolepsia em um modelo canino ${ }^{8}$. As hipocretinas/orexinas são novos neuropeptídeos hipotalâmicos envolvidos nas funções neuroendócrina e de homeostase de energia ${ }^{10}$. A deficiência de ligação de hipocretina é observada em muitos pacientes narcolépticos com cataplexia ${ }^{11,12}$. Já que a hipocretina geralmente participa da manutenção da vigília, a perda dos neurônios que liberam este peptídeo pode permitir que o sono REM ocorra a qualquer momento e determine os ataques catalépticos ${ }^{9}$. Estes achados permitem uma nova compreensão da patofisiologia da narcolepsia: uma característica genética específica (a mutação de HLA no cromossomo 6) pode aumentar a susceptibilidade dos neurônios que contêm hipocretina a ataques imunes. A hipótese mais provável para a narcolepsia é um processo auto-imune que atinge os neurônios de hipocretina em resposta a fatores ambientais desconhecidos 9-11,13,14. Dentro desta perspectiva, os neurologistas e pediatras devem estar cientes que o tratamento inicial com imunoglobulinas em crianças e adolescentes recém-diagnosticados com narcolepsia pode atrasar o avanço da doença e até mesmo limitar ou evitar a necessidade de tratamento crônico com anfetaminas, agentes promotores de vigília (modafinil) ou antidepressivos ${ }^{15}$.

Recentemente, foi proposto que a regulação negativa do gene $M \times 2$ nas células brancas do sangue de pacientes narcolépticos e a expressão do gene NLC1-A em uma região específica do cromossomo 21 (21q22.3) são novos fatores genéticos relacionados com a narcolepsia ${ }^{16,17}$. No entanto, o gene DQB1*0602 continua sendo o melhor marcador de HLA para todos os grupos étnicos ${ }^{8,18}$.

\section{Síndrome do avanço da fase do sono e síndrome do atraso da fase do sono}

O sono é regulado por um processo homeostático que determina sua necessidade e por um processo circadiano que determina sua freqüência ${ }^{19}$. O ciclo vigília-sono é controlado pelo relógio circadiano, e o primeiro gene do relógio a ser isolado (Clock, per) foi identificado em uma mosca drosophila. Mais tarde, contrapartes homólogas foram encontradas em mamíferos ${ }^{20}$.

Recentemente, dois genes envolvidos em distúrbios circadianos do sono foram descobertos ${ }^{21}$. A síndrome do atraso de fase do sono (SAFS) é caracterizada pelo atraso do início do sono e pela demora para acordar (3-6 horas) em relação aos horários convencionais para as duas ações. Os pacientes típicos normalmente não adormecem antes das $2 \mathrm{~h}$ da manhã e não acordam antes das 10-12h, apesar da arquitetura do sono poder ser considerada normal. Os pacientes normalmente relatam estarem mais alertas durante a noite do que durante a manhã. Quando o paciente é forçado a acordar mais cedo devido a compromissos escolares, o resultado pode ser a insuficiência crônica de sono e o excesso de sonolência diurna. Esta é uma das reclamações mais freqüentes na adolescência, associada também a problemas de desempenho escolar $^{1,20}$. O atraso de fase de sono está associado ao polimorfismo em Per3. A síndrome do avanço de fase do sono é caracterizada por horários de início de sono e de acordar habitual e involuntariamente mais cedo do que a média da sociedade. Esta síndrome, menos comum do que a SAFS, geralmente ocorre na faixa etária pediátrica ${ }^{19}$ e é causada por uma mutação pontual em um gene do relógio biológico $($ Per2) 21 . 


\section{Insônia}

A insônia é definida como a dificuldade iniciar e/ou manter o sono. A insônia infantil geralmente está relacionada com uma série de fatores externos e ambientais ou a hábitos que variam com a idade, da primeira infância à adolescência. Sua patofisiologia é completamente diferente na idade adulta e, portanto, a abordagem clínica do pediatra também deve ser ${ }^{22}$. Apesar dessa queixa estar associada freqüentemente a história familiar positiva, nenhum gene específico foi descoberto para a insônia.

Pesquisadores usaram modelos animais de privação do sono para identificar o gene Homer1a, cuja expressão reflete a susceptibilidade à perda de sono ${ }^{19}$. Pacientes adultos homozigóticos para o genótipo do gene clock estão mais predispostos à insônia inicial, média e terminal. Além disso, a insônia familiar fatal, uma doença letal que não ocorre na infância, foi relacionada com uma mutação pontual no gene da proteína priônica ${ }^{21}$.

\section{Apnéia obstrutiva do sono}

Em crianças, muitas reclamações e síndromes diferentes estão relacionadas com a apnéia do obstrutiva do sono ${ }^{23}$. A presença de alterações sutis no sono, medidas pelo padrão alternante cíclico em crianças com apnéias obstrutivas, já foi relatada anteriormente ${ }^{24}$.

A etiologia da apnéia do obstrutiva do sono é multifatorial e consiste de uma associação entre fatores anatômicos e neuromusculares, além da predisposição genética. A obesidade relacionada com a apnéia do obstrutiva do sono atinge níveis de problema de saúde pública entre crianças, principalmente em países desenvolvidos ${ }^{25}$.

Dados recentes indicam que a apnéia do obstrutiva do sono em adultos pode ser influenciada por fatores genéticos relativos à obesidade e distribuição de gordura corporal, controle muscular das vias respiratórias superiores, morfologia craniofacial (redução no tamanho mandibular e das vias respiratórias), controle respiratório e sono. Além disso, alguns polimorfismos genéticos foram identificados nesta população, mas ainda não se sabe se os genes candidatos reconhecidos são diretamente causais para o fenótipo ou não ${ }^{25-28}$. Estudos com roedores sugerem que a deficiência de monoaminoxidase $A$ associada a aumentos na apnéia do sono e excesso de serotonina podem contribuir para este fenótipo ${ }^{29}$.

Um estudo recente publicou a hipótese que a apnéia do obstrutiva do sono pediátrica levaria a uma alteração na expressão genética em leucócitos circulantes. Os resultados mostram que o RNA derivado de leucócitos periféricos confirma a presença de expressão alterada de agrupamentos genéticos funcionalmente relevantes na apnéia do obstrutiva do sono pediátrica ${ }^{30}$.

Sabe-se que a gravidade da apnéia do obstrutiva do sono representa apenas aproximadamente $40 \%$ da variância em desempenho cognitivo, e que os determinantes genéticos da susceptibilidade individual podem contribuir para a morbidade. Recentemente, Gozal et al. ${ }^{31}$ demonstraram que o alelo apolipoproteína $E$ (ApoE) epsilon4 estava presente em três de 199 crianças sem ASO, ao passo que naquelas com ASO, o mesmo alelo estava presente em 16 de 146 crianças ( $p<$ 0,0002 ), e que tais resultados também estavam correlacionados com os resultados de testes cognitivos. Os resultados sugerem que o alelo ApoE epsilon4 não está associado apenas a um risco maior de distúrbios respiratórios do sono, mas também a riscos maiores de disfunção neurocognitiva ${ }^{31}$. A apnéia do sono é uma doença complexa, e a identificação dos loci genéticos de susceptibilidade fortalecerão nossa capacidade de avaliar o risco ${ }^{29-33}$. A identificação dos genes relacionados com a patogênese desta síndrome pode levar ao desenvolvimento de agentes terapêuticos que modifiquem seu curso natural e previnam a morbidade cardiovascular e neurocognitiva em crianças $^{28}$.

\section{Síndrome de hipoventilação central congênita}

A síndrome de hipoventilação central congênita é um raro distúrbio do controle respiratório caracterizado pela hipoventilação durante o sono, a não-responsividade a estimulantes respiratórios e a inconsciência da hipóxia com respiração básica adequada durante a vigília. O curso clínico varia, mas os sintomas podem ser reconhecidos, em alguns casos, desde os primeiros dias de vida (cianose, apnéia, falhas respiratórias súbitas durante o sono). A doença costumava ser conhecida como maldição de Ondina ${ }^{34}$. A síndrome pode ocorrer em combinação com outras manifestações clínicas , como a síndrome de Moebius. Mais recentemente, alguns pesquisadores relataram uma forte associação com a doença de Hirschprung e tumores de origem na crista neural ${ }^{35,36}$. Os primeiros casos desta associação foram informados por Haddad e colaboradores em $1978^{37}$. O termo "neurocristopatia", que inclui este diagnóstico, foi proposto em $1980^{38}$. Cerca de $50 \%$ dos pacientes com síndrome de hipoventilação central congênita apresentam doença de Hirschprung, e cerca de $20 \%$ desenvolvem tumores da crista neural. ${ }^{35}$. A doença resulta de uma mutação (expansão de repetições de polialanina) do gene homeobox (PHOX) 2B ou PHOX2B, que é crítico para o desenvolvimento da maioria dos transmissores do sistema nervoso autônomo, incluindo todos os derivativos da crista neural autônoma ${ }^{39}$. A penetração incompleta desta alteração gênica pode ocorrer em famílias; portanto, a intensidade dos sintomas pode variar. Indica-se a realização de uma avaliação genética em familiares de casos afetados para se estabelecer o risco reprodutivo e de desenvolvimento de hipoventilação alveolar ${ }^{40,41}$.

\section{Síndrome da morte súbita do lactente}

A síndrome da morte súbita do lactente (SMSL) é definida como "a morte súbita de um lactente de menos de um ano de idade que continua inexplicada após uma investigação completa do caso, incluindo a realização de uma autópsia completa, análise da cena da morte e revisão do histórico clínico"42. Fatores de risco comportamentais foram identificados, e os mais prevalentes são o uso da posição prona, a 
exposição ao tabaco, camas macias e excesso de calor. Os irmãos de vítimas de SMSL correm risco maior de SMSL. No entanto, em casos familiares, distúrbios metabólicos ou genéticos como a síndrome do QT longo e erros inatos do metabolismo devem ser excluídos ${ }^{43-46}$.

Até o momento, não foi identificada nenhuma diferença genotípica que poderia ser ligada aos fenótipos clinicamente definidos. No entanto, diversos polimorfismos diferentes (canais de sódio e potássio, transportadores de serotonina, em genes envolvidos no desenvolvimento do sistema nervoso autônomo, tais como PHOX2a, RET, ECE1, TLX3 e EN1, e em citocina-interleucina 10 - IL10) foram relatados quando em comparações entre SMSL e controles ${ }^{46}$.

\section{Síndrome das pernas inquietas}

A SPI é um distúrbio do sono cujo diagnóstico muitas vezes é confundido com dores do crescimento na infância ${ }^{1}$. A SPI é caracterizada por uma ânsia irresistível e constante por mover as pernas, mesmo quando a pessoa está adormecendo, e por sensações desconfortáveis nos membros inferiores. A SPI é uma causa importante de interrupções do sono, e na maioria dos pacientes é possível detectar movimentos periódicos dos membros durante o sono ${ }^{47-50}$. No entanto, o papel do movimento periódico dos membros na produção de sonolência é controverso, e algumas publicações recentes sugerem que são apenas marcadores de despertares relacionados com esforços respiratórios ${ }^{51}$. A patogênese da SPI também já foi associada à deficiência de ferro. Em um estudo populacional realizado recentemente na Islândia, uma variante comum em um íntron de BTBD9 no cromossomo 6p21.2 foi observada como marcador de movimentos periódicos dos membros que ocorre em forte associação com níveis séricos reduzidos de ferritina $^{52}$. Em um estudo alemão, foram observadas associações significativas entre a SPI e variantes intrônicas no gene homeobox MEIS1, BTBD9, MAP2K5 e o fator de transcrição LBXCOR1 nos cromossomos 2p, 6p e 15q, respectivamente. Como o gene MEIS1 está implicado no desenvolvimento dos membros, este achado levanta a possibilidade da SPI ser um distúrbio desenvolvimental ${ }^{53}$.

\section{Parassônias}

As parassônias pediátricas reúnem uma variedade de comportamentos e atividade motora alterados durante o sono. A American Academy of Sleep Medicine (Academia Americana de Medicina do Sono) define as parassônias como "eventos ou experiências físicas indesejáveis que ocorrem no início do sono, durante o sono ou ao despertar". Estes eventos incluem movimentos, comportamentos, emoções, percepções, sonhos e funcionamento do sistema nervoso autônomo anormais relativos ao sono ${ }^{1}$.

Nesta revisão, consideramos apenas aquelas parassônias infantis para as quais existe uma possível base genética.

\section{Sonambulismo, despertar confusional e terror noturno}

O sonambulismo, o despertar confusional e o terror noturno são parassônias que ocorrem durante o sono calmo (NREM) e freqüentemente compartilham de um histórico familiar positivo ${ }^{1,21}$. Nenhum modo de transmissão genético foi identificado até o momento, mas a alta concordância entre gêmeos homozigóticos e uma associação com o alelo HLA (DQB1*0501) na forma familiar foram identificadas ${ }^{54}$. Também já foi relatada uma predisposição genética comum (DQB1*05) entre sonambulismo e distúrbio de comportamento do sono REM ${ }^{55}$.

\section{Enurese noturna}

A enurese noturna é considerada um dos problemas do sono mais prevalentes e persistentes na infância. A enurese noturna é definida como dois ou mais episódios de incontinência urinária em um mês para crianças entre 5 e 6 anos de idade, e um ou mais episódios após os 6 anos de idade na ausência de distúrbios físicos como diabete, convulsões ou infecções do trato urinário ${ }^{1}$. O histórico familiar é a variável etiológica de apoio mais consistente. A enurese é transmitida mais freqüentemente na forma de herança autossômica dominante, com alta penetração (90\%). Quatro loci genéticos foram identificados $(8 q, 12 q, 12 q, 22 q 11)^{56}$.

\section{Conclusões}

Apesar da maioria dos distúrbios do sono ainda não terem uma base molecular identificada, técnicas modernas são cada vez mais usadas para determinar a contribuição dos genes ao sono e aos seus distúrbios associados. A importância clínica destas descobertas pode estar relacionada com a melhoria de métodos diagnósticos, mas também como alvo para o desenvolvimento de medicações.

\section{Referências}

1. Nunes ML. Sleep disorders. J Pediatr (Rio J). 2002;78 Suppl $1:$ S63-72.

2. Tafti M, Dauvilliers $Y$, Overeem S. Narcolepsy and familial advanced sleep-phase syndrome: molecular genetics of sleep disorders. Curr Opin Genet Dev. 2007;17:222-7.

3. Bruni O, Ferini-Strambi L, Russo PM, Antignani M, Innocenzi $M$, Ottaviano $\mathrm{P}$, et al. Sleep disturbances and teacher ratings of school achievement and temperament in children. Sleep Med. 2006; 7:43-8.

4. Tafti M. Quantitative genetics of sleep in inbred mice. Dialogues Clin Neurosci. 2007; 9:273-8.

5. Mackiewicz M, Shockley KR, Romer MA, Galante RJ, Zimmerman JE, Naidoo N, et al. Macromolecule biosynthesis: a key function of sleep. Physiol Genomics. 2007;31:441-57.

6. Gottlieb DJ, O'Connor GT, Wilk JB. Genome-wide association of sleep and circadian phenotypes. BMC Med Genet. 2007; 8 Suppl 1:S9.

7. Miyazaki K, Wakabayashi M, Chikahisa S, Sei H, Ishida N. PER2 controls circadian periods through nuclear localization in the suprachiasmatic nucleus. Genes Cells. 2007;12:1225-34.

8. Guilleminaut C, Anagnos A. Narcolepsy. In: Kryger MH, Roth T, Dement $C$, editors. Principles and practice of sleep medicine. Philadelphia: WB Saunders; 2000. p. 676-92.

9. Wurtman RJ. Narcolepsy and the hypocretins. Metabolism. 2006; 55: S36-9. 
10. Ganjavi H, Shapiro CM. Hypocretin/orexin: a molecular link between sleep, energy regulation and pleasure. J Neuropsychiatry Clin Neurosci. 2007;19:413-9.

11. Nishino S. Clinical and neurobiological aspects of narcolepsy. Sleep Med. 2007;8:373-99.

12. Heier MS, Evsiukova T, Vilming S, Gjerstad MD, Schrader $H$, Gautvik K. CSF hypocretin-1 levels and clinical profiles in narcolepsy and idiophatic CNS hypersomnia in Norway. Sleep. 2007;30:969-73.

13. Dauvilliers $Y$, Tafti M. Molecular genetics and treatment of narcolepsy. Ann Med. 2006;38:252-62.

14. Taheri S. The genetics of sleep disorders. Minerva Med. 2004; 95:203-12.

15. Dauvilliers Y. Follow-up of four narcolepsy patients treated with intravenous immunoglobulins. Ann Neurol. 2006;60:153.

16. Tanaka S, Honda Y, Honda M. Identification of differentially expressed genes in blood cells of narcoleptic patients. Sleep. 2007;30:974-9.

17. Kawashima M, Tamiya G, Oka A, Hohjoh H, Juji T, Ebisawa T, et al. Genomewide association analysis of human narcolepsy and a new resistance gene. Am J Hum Genet. 2006; 79:252-63

18. Pedrazzoli M, Pontes JC, Peirano P, Tufik S. HLA-DQB1 genotyping in a family with narcolepsy-cataplexy. Brain Res. 2007;1165:1-4.

19. Maret S, Dorsaz S, Gurcel L, Pradervand S, Petit B, Pfister C, et al. Homer1a is a core brain molecular correlate of sleep loss. Proc Natl Acad Sci USA. 2007;104:20090-5.

20. Baker SK, Zee PC. Circadian disorders of the sleep-wake cycle. In: Kryger MH, Roth T, Dement C, editors. Principles and practice of sleep medicine. Philadelphia: WB Saunders; 2000. p. 606-14.

21. Hamet $P$, Tremblay J. Genetics of the sleep-wake cycle and its disorders. Metabolism. 2006;55:S7-12.

22. Nunes $M L$, Cavalcante V. Clinical evaluation and treatment of insomnia in childhood. J Pediatr (Rio J). 2005;81:277-86.

23. Guilleminaut C, Lee JH, Chan A. Pediatric obstructive sleep apnea syndrome. Arch Pediatr Adolesc Med. 2005;159:775-85.

24. Kheirandish-Gozal L, Miano S, Bruni O, Ferri R, Pagani J, Villa MP, et al. Reduced NREM sleep instability in children with sleep disordered breathing. Sleep. 2007;30:450-7.

25. Kalra M, Chakraborty R. Genetic susceptibility to obstructive sleep apnea in the obese children. Sleep Med. 2007;8:169-75.

26. Schwab RJ. Genetic determinants of upper airway structures that predispose to obstructive sleep apnea. Respir Physiol Neurobiol. 2005;147:289-98.

27. Tung A. The biology and genetics of obesity and obstructive sleep apnea. Anesthesiol Clin North America. 2005;23:445-61.

28. Kaparianos A, Sampsonas F, Karkoulias K, Spiropoulos K. Obstructive sleep apnoea syndrome and genes. Neth J Med. $2006 ; 64: 280-9$.

29. Real C, Popa D, Seif I, Callebert J, Launay JM, Adrien J, et al. Sleep apneas are increased in mice lacking monoamine oxidase A. Sleep. 2007;30:1295-302.

30. Khalyfa A, Capdevila OS, Buazza MO, Serpero LD, Kheirandish-Gozal L, Gozal D. Genome-wide gene expression profiling in children with non-obese obstructive sleep apnea. Sleep Med. In press 2008.

31. Gozal D, Capdevila OS, Kheirandish-Gozal L, Crabtree VM. APOE epsilon 4 allele, cognitive dysfunction, and obstructive sleep apnea in children. Neurology. 2007;69:243-9.
32. Raizen DM, Mason TB, Pack AI. Genetic basis for sleep regulation and sleep disorders. Semin Neurol. 2006;26:467-83.

33. Polotsky VY, O'Donnell CP. Genomics of sleep-disordered breathing. Proc Am Thorac Soc. 2007;4:121-6.

34. Nunes ML, Friedrich MG, Loch LF. Association of misoprostol, Moebius syndrome and congenital central alveolar hypoventilation. Case report. Arq Neuropsiquiatr. 1999; 57:88-91.

35. Nunes $\mathrm{ML}$, Fiori $\mathrm{HH}$, Holzhey $\mathrm{C}$. Neurochristopathy in the differential diagnosis of newborn's apnea: case report. Arq Neuropsiquiatr. 2001;59:968-71.

36. Parodi S, Bachetti T, Lantieri F, Di Duca M, Santamaria G, Ottonello G, et al. Parental origin and somatic mosaicism of PHOX2B mutations in congenital central hypoventilation syndrome. Hum Mutat. 2008;29:206.

37. Haddad GG, Mezza NM, Defendini R, Blanc WA, Driscoll JM, Epstein MA, et al. Congenital failure of automatic control of ventilation, gastrointestinal motility and heart rate. Medicine (Baltimore). 1978;57:517-26.

38. Bower RJ, Adkins JC. Ondine's curse and neurocristopathy. Clin Pediatr (Phila). 1980;19:665-8.

39. Gaultier C, Trang H, Dauger S, Gallego J. Pediatric disorders with autonomic dysfunction: what role for PHOX2B? Pediatr Res. 2005;58:1-6.

40. Berry-Kravis EM, Zhou L, Rand CM, Weese-Mayer DE. Congenital central hypoventilation syndrome: $\mathrm{PHOX} 2 \mathrm{~B}$ mutations and phenotype. Am J Respir Crit Care Med. 2006; 174:1139-44.

41. Doherty LS, Kiely JL, Deegan PC, Nolan G, McCabe S, Green AJ, et al. Late-onset central hypoventilation syndrome : a family genetic study. Eur Respir J. 2007;29:312-6.

42. Willinger M, James LS, Catz C.Defining the sudden death syndrome (SIDS): deliberations of an expert panel convened by the National Institute of child Health and Human Development. Pediatr Pathol. 1991;11:677-84.

43. Geib LT, Aerts D, Nunes ML. Sleep practices and sudden death syndrome: a new proposal for scoring risk factors. Sleep. 2006; 29:1288-94.

44. Geib LT, Nunes ML. The incidence of sudden infant death syndrome in a cohort of infants. J Pediatr (Rio J). 2006;82:21-6

45. Geib LT, Nunes ML. Sleeping habits related to sudden infant death syndrome: a population based study. Cad Saude Publica. 2006;22:415-23.

46. Moon RY, Horne RS, Hauck FR. Sudden infant death syndrome. Lancet. 2007;370:1578-87.

47. Zucconi M, Ferri R, Allen R, Baier PC, Bruni O, Chokroverty S, et al.; International Restless Legs Syndrome Study Group (IRLSSG). The official World Association of Sleep Medicine (WASM) standards for recording and scoring periodic leg movements in sleep (PLMS) and wakefulness (PLMW) developed in collaboration with a task force from the International Restless Legs Syndrome Study Group (IRLSSG). Sleep Med. 2006; 7:175-83.

48. Ferri R, Zucconi M, Manconi M, Plazzi G, Bruni O, Ferini-Strambi L. New approaches to the study of periodic leg movements during sleep in restless legs syndrome. Sleep. 2006; 29:759-69.

49. Ferri R, Manconi M, Lanuzza B, Cosentino FI, Bruni O, Ferini-Strambi $L$, et al. Age-related changes in periodic legs movements during sleep in patients with restless legs syndrome. Sleep Med. In press 2007. 
50. Ferri R, Zucconi M, Manconi M, Bruni O, Ferini-Strambi L, Vandi $S$, et al. Different periodicity and time structure of leg movements during sleep in narcolepsy/cataplexy and restless legs syndrome. Sleep. 2006;29:1587-94.

51. Patel S. Restless legs syndrome and periodic limb movements of sleep: fact, fad, and fiction. Curr Opin Pulm Med. 2002; 8:498-501.

52. Stefansson $H$, Rye DB, Hicks A, Petursson $H$, Ingason $A$, Thorgeirsson TE, et al. A genetic risk factor for periodic limb movement. N Engl J Med. 2007;357:639-47.

53. Winkelmann J, Schormair B, Lichtner P, Ripke S, Xiong L, Jalilzadeh S, et al. Genome-wide association study of restless legs syndrome identifies common variants in three genomic regions. Nat Genet. 2007;39:1000-6.

54. Lecendreux M, Bassetti C, Dauvilliers Y, Mayer G, Neidhart E, Tafti M. HLA and genetic susceptibility of sleep walking. Mol Psychiatr. 2003;8:114-7.
55. Schenck CH, Boyd JL, Mahowald MV. A parasomnia overlap disorders involving sleepwalking, sleep terrors and REM sleep behavior disorder in 33 polysomnographically confirmed cases. Sleep. 1997;20:972-81.

56. Dauvilliers $Y$, Maret S, Tafti M. Genetics of normal and pathological sleep in humans. Sleep Med Rev. 2005;9:91-100.

Correspondência:

Magda Lahorgue Nunes

Serviço de Neurologia, Hospital São Lucas da PUCRS

Av. Ipiranga, 6690, sala 220

CEP 90610-000 - Porto Alegre, RS

Tel.: (51) 3339.4936

E-mail: nunes@pucrs.br 